\title{
Neutron reactions in massive stars and the origin of ${ }^{60} \mathrm{Fe}$
}

\author{
F. Käppeler*, I. Dillmann; C. Domingo Pardo J. Marganiec\$, and S. Walter \\ Forschungszentrum Karlsruhe, Institut für Kernphysik, 76344 Eggenstein-Leopoldshafen, \\ Germany \\ E-mail: franz.kaeppeler@ik.fzk.de \\ E. Uberseder, J. Görres, M. Wiescher \\ University of Notre Dame, Department of Physics, Notre Dame, Indiana, USA
}

M. Heil, R. Reifarth

GSI Darmstadt, Planckstr. 1, 64291 Darmstadt, Germany

\section{Schumann, J. Neuhausen}

Laboratory for Environmental and Radiochemistry, PSI, 5232 Villigen PSI, Switzerland

\section{Pignatari}

Keele University, Keele, Staffordshire ST5 5BG, UK

\begin{abstract}
Slow neutron capture reactions in massive stars are substantially contributing to the abundances of the elements between Fe and $\mathrm{Zr}$, producing the so-called weak $s$ component. The weak $s$ process is characterized by a low neutron exposure, where the reaction flow does not reach equilibrium conditions. As a consequence, uncertainties in the stellar $(n, \gamma)$ rates give rise to large propagation effects. This is illustrated by the impact of recently improved experimental cross sections. Massive stars are also the main production site of the unstable isotope ${ }^{60} \mathrm{Fe}$, which provides an important chronometer for the early solar system. The successful measurement of the stellar $(n, \gamma)$ cross section of ${ }^{60} \mathrm{Fe}$ represents an important step towards a quantitative determination of the $s$-process origin of ${ }^{60} \mathrm{Fe}$ in massive stars.
\end{abstract}

10th Symposium on Nuclei in the Cosmos

July 27 - August 1, 2008

Mackinac Island, Michigan, USA

\footnotetext{
* Speaker.

†present address: Physik-Department E12, Technische Universität München, Garching, Germany

†present address: GSI Darmstadt, Planckstr. 1, 64291 Darmstadt, Germany

$\S$ present address: Division of Nuclear Physics, University of Lodz, Pomorska 149/153, 90-236 Lodz, Poland
} 


\section{The $s$ process in massive stars}

The weak $s$ process contributes to the solar abundance distribution in the mass range $A \leq 90$ during the late evolutionary stages of stars with $M \geq 8 M_{\odot}\left(M_{\odot}\right.$ stands for the mass of the sun). Due to the comparably short lifetimes of massive stars, the production of $s$-process material starts with the lighter $s$ elements, an important aspect of Galactic chemical evolution.

Stellar models of these massive stars have shown that the weak $s$ process operates in two steps, during convective core He burning and subsequently during convective shell carbon burning. Depending on the stellar model an additional contribution to the final $s$ yields may result from a small convective He burning layer at the outer border of the $\mathrm{C}$ shell $[1,2]$. Neutrons are mainly produced by the ${ }^{22} \mathrm{Ne}(\alpha, n){ }^{25} \mathrm{Mg}$ reaction. During core He burning this occurs near core He exhaustion at temperatures of $T=(2.5-3.5) \times 10^{8} \mathrm{~K}$ and low neutron densities of $\leq 10^{6} \mathrm{~cm}^{-3}$. During the subsequent carbon shell burning phase neutron densities with peak values of about $10^{2} \mathrm{~cm}^{-3}$ are reached due to the higher temperatures of $(1.0-1.4) \times 10^{9} \mathrm{~K}[3,4,5]$.

At present, the main features of the weak $s$ process in massive stars are quite well understood. However, a good knowledge of the stellar $(n, \gamma)$ cross sections of the involved isotopes are extremely important for a detailed analysis of the $s$-process distribution and for a comparison with the solar system abundances.

Cross sections are not only influencing the abundance of a particular isotope but have a potentially strong propagation effect on the abundances of the subsequent isotopes in the s-process chain as demonstrated at the example the ${ }^{62} \mathrm{Ni}(n, \gamma){ }^{63} \mathrm{Ni}$ reaction [1]. In fact, several isotopes between iron and strontium exhibit sufficiently small neutron capture cross section (lower than $\sim 50-100$ mbarn) to act as bottle-necks for the $s$-process reaction flow.

With very few exceptions, however, the necessary accuracy of stellar $(n, \gamma)$ data has not yet been achieved in the mass region of the weak $s$ process. Existing data suffer not only from large uncertainties of more than $10 \%$, but exhibit discrepancies, which often exceed the quoted uncertainties considerably [7]. Therefore, a series of activation measurements on $\mathrm{Fe}, \mathrm{Co}$, and $\mathrm{Ni}$ isotopes, all situated in or near the Fe abundance peak, have been performed by means of the activation technique. Since these isotopes have relatively small Maxwellian averaged cross sections (MACS), they are expected to give rise to large propagation effects in the final abundance distribution. The experimental technique and the measurements are described in Sec. II. Sec. III deals with the case of the unstable isotope ${ }^{60} \mathrm{Fe}\left(t_{1 / 2}=1.5 \times 10^{6} \mathrm{yr}\right)$, which is of great interest for the related chronometric aspects.

\section{Activation measurements between $\mathrm{Fe}$ and $\mathrm{Ni}$}

\subsection{Experimental technique}

The activation method represents a well established and accurate approach to determine MACSs at $k T=25 \mathrm{keV}$ by producing a quasi-stellar neutron spectrum via the ${ }^{7} \operatorname{Li}(p, n)^{7} \mathrm{Be}$ reaction $[8,9]$. With the proton beam energy adjusted to $E_{p}=1912 \mathrm{keV}, 30 \mathrm{keV}$ above the reaction threshold, all neutrons emitted from a $30 \mu \mathrm{m}$ thick metallic Li production target are kinematically collimated into a forward cone of $120^{\circ}$ opening angle. Target cooling is achieved by lateral heat conduction 


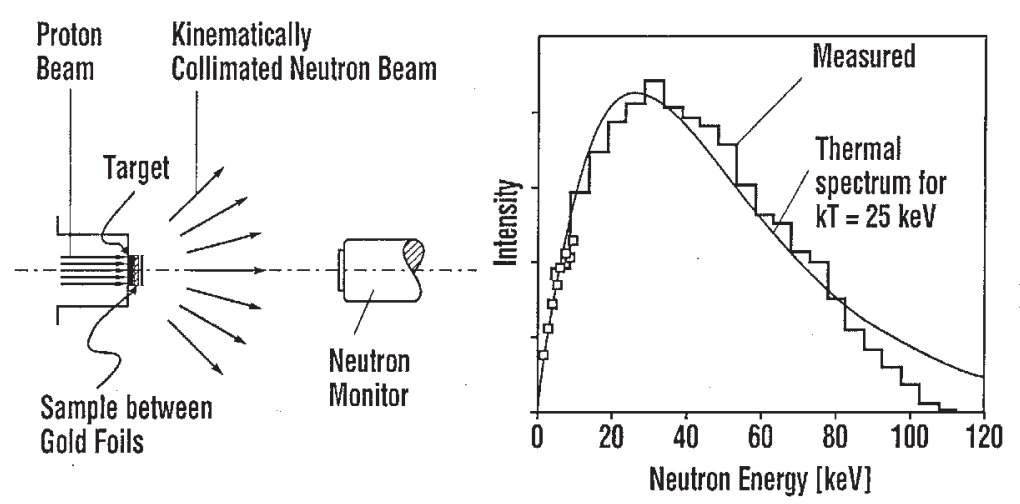

Figure 1: Left: Schematic sketch of the activation setup. Right: The quasi-stellar neutron spectrum obtained with the ${ }^{7} \mathrm{Li}(p, n)^{7} \mathrm{Be}$ reaction (histogram and symbols) compared to a true Maxwell-Boltzmann distribution for a thermal energy of $k T=25 \mathrm{keV}$. Note that the experimental spectrum is truncated at $106 \mathrm{keV}$.

to the water flow outside of this cone, thus avoiding neutron moderation. Throughout the irradiations the neutron flux was continuously monitored and recorded in time steps of typically $60 \mathrm{~s}$ by means of a ${ }^{6} \mathrm{Li}$-glass detector at $1 \mathrm{~m}$ distance from the target. A sketch of the experimental setup and a comparison of the resulting quasi-stellar neutron spectrum with a true Maxwell-Boltzmann distribution are shown in Fig. 1.

The samples were made from metal foils of natural composition with different dimensions (6, 8,10 , and $12 \mathrm{~mm}$ in diameter and between 0.03 and $0.5 \mathrm{~mm}$ in thickness) in order to check for systematic uncertainties. During the activations, the samples were sandwiched between $0.03 \mathrm{~mm}$ thick gold foils for normalization to the well known gold reference cross section [9].

Depending on the half-lives of the respective product nuclei, the irradiations lasted between $115 \mathrm{~h}$ in case of ${ }^{58} \mathrm{Fe}$ and $15 \mathrm{~min}$ in case of ${ }^{65} \mathrm{Cu}$ with neutron production rates of $(0.7-3.0) \times 10^{9}$ $\mathrm{s}^{-1}$. In total, 14 activations have been carried out for the redundant determination of the $(n, \gamma)$ cross sections of ${ }^{58} \mathrm{Fe},{ }^{59} \mathrm{Co},{ }^{64} \mathrm{Ni},{ }^{63} \mathrm{Cu}$, and ${ }^{65} \mathrm{Cu}$.

The induced activities are characterized by energetic $\gamma$-ray lines with well known relative intensities. The $\gamma$-ray counting was performed by means of a shielded $76 \mathrm{~cm}^{3} \mathrm{HPGe}$-detector with $1.7 \mathrm{keV}$ resolution at $1.33 \mathrm{MeV} \gamma$-ray energy and a relative efficiency of $30 \%$. Only two of the irradiated Ni samples were counted with a HPGe clover detector of $120 \%$ relative efficiency to improve the statistical uncertainty. Special adapters were used to ensure that the positioning of the samples was reproducible within $0.1 \mathrm{~mm}$. In all cases $\gamma$-ray backgrounds were small and did not affect the uncertainty of the final cross sections. The excellent sensitivity of the experimental method is illustrated in Fig. 2 by the most unfavorable case of the ${ }^{59} \operatorname{Co}(n, \gamma){ }^{60} \mathrm{Co}$ reaction. Although the induced activities were low because of the long half-life of ${ }^{60} \mathrm{Co}\left(t_{1 / 2}=5.27 \mathrm{yr}\right)$, the statistical uncertainties were much smaller than the respective systematic effects. 


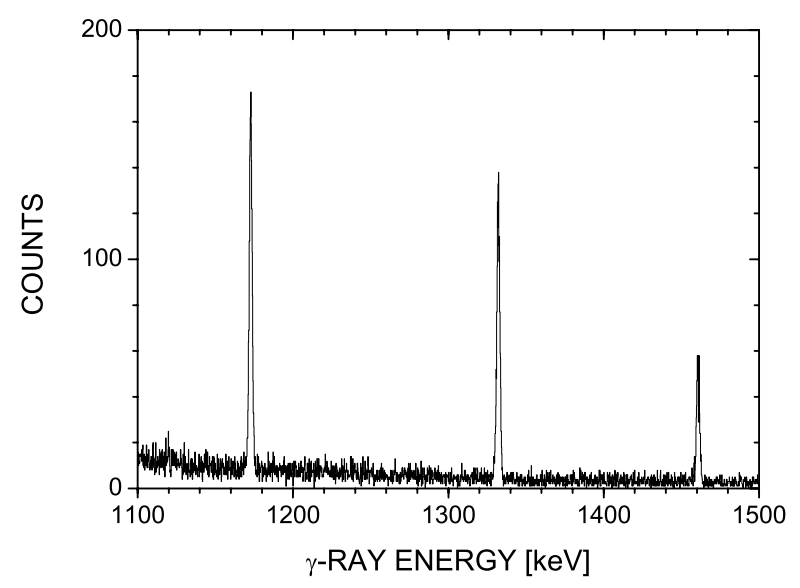

Figure 2: The $\gamma$-ray spectrum corresponding to the most unfavorable case of the ${ }^{59} \mathrm{Co}(n, \gamma){ }^{60} \mathrm{Co}$ reaction, where the long half-life of ${ }^{60} \mathrm{Co}$ results in a low signal/background ratio. Nevertheless, the lines at 1173 and $1332 \mathrm{keV}$ could be analyzed with sufficient statistical accuracy. The background peak on the right corresponds to the ${ }^{40} \mathrm{~K}$ line at $1461 \mathrm{keV}$.

\subsection{Results at $k T=25 \mathrm{keV}$ and extrapolation to higher temperatures}

The total number of activated nuclei $\mathrm{A}$ is given by

$$
A=\phi N \sigma f_{b}
$$

where $\phi$ is the time integrated neutron flux, $N$ the number of sample atoms, and $\sigma$ the spectrum averaged neutron capture cross section. The factor $f_{b}$ accounts for variations of the neutron flux and for the decay during activation. The number of counts in a characteristic $\gamma$-ray line is

$$
C_{\gamma}=A K_{\gamma} \varepsilon_{\gamma} I_{\gamma} f_{w} f_{m}
$$

where $K_{\gamma}$ is a correction factor for $\gamma$-ray self-absorption, summing, and extended source effects, $\varepsilon_{\gamma}$ the detection efficiency of the HPGe detector, and $I_{\gamma}$ the line intensity. The time factors $f_{w}=e^{-\lambda t_{w}}$ and $f_{m}=\left(1-e^{-\lambda t_{m}}\right)$ account for the fraction of nuclei that decay during the waiting time between activation and counting and during the measurement itself. The time-integrated flux per activation, $\phi$, is determined from the intensities of the $412 \mathrm{keV} \gamma$-ray line in the spectra of the two gold foils.

In total, 14 activations were carried out to determine the five investigated cross sections. In spite of the different experimental parameters used, the results were found to be consistent within the estimated uncertainties, thus confirming the procedures applied in data analysis. The final uncertainties between 3 and 5\% are mostly determined by the gold reference cross section, the efficiency of the HPGe detectors, and the time integrated neutron flux. In all cases, the uncertainties of previous measurements could be improved by factors of 2 to 4 .

The measured values represent averaged cross sections for the experimental neutron distribution used in the irradiations. Though the experimental spectrum corresponds in very good approximation to the thermal spectrum for $k T=25 \mathrm{keV}$, the cutoff at $106 \mathrm{keV}$ requires a small correction, 
Table 1: Maxwellian averaged $(n, \gamma)$ cross sections (in mbarn) for $k T=30 \mathrm{keV}$.

\begin{tabular}{lcc}
\hline Isotope & Bao et al. [7] & New results [11] \\
\hline${ }^{58} \mathrm{Fe}$ & $12.1 \pm 1.3$ & $13.5 \pm 0.7$ \\
${ }^{59} \mathrm{Co}$ & $38 \pm 4$ & $39.6 \pm 2.6$ \\
${ }^{64} \mathrm{Ni}$ & $8.7 \pm 0.9$ & $8.0 \pm 0.6$ \\
${ }^{63} \mathrm{Cu}$ & $94 \pm 10$ & $56.0 \pm 3.5$ \\
${ }^{65} \mathrm{Cu}$ & $41 \pm 5$ & $30.0 \pm 1.6$ \\
\hline
\end{tabular}

in particular if the investigated cross section exhibits a different energy dependence than the gold reference cross section. The final MACS is

$$
\frac{<\sigma \mathrm{v}>}{\mathrm{v}_{T}}=\frac{2}{\sqrt{\pi}} \frac{\int_{0}^{\infty} \sigma\left(E_{n}\right) \cdot E_{n} \cdot \exp \left(-E_{n} / k T\right) \cdot d E_{n}}{\int_{0}^{\infty} E_{n} \cdot \exp \left(-E_{n} / k T\right) \cdot d E_{n}}
$$

where $\sigma\left(E_{n}\right)$ is the energy differential $(n, \gamma)$ cross section obtained by means of the Hauser-Feshbach statistical model [10], normalized to the measured value by folding with the experimental neutron spectrum. By this procedure the slight differences between the experimental spectrum and a true Maxwell-Boltzmann distribution at $k T=25 \mathrm{keV}$ are taken into account. The normalized cross section can also be used to derive the MACS at other temperatures of astrophysical interest. However, such an extrapolation may well be affected by additional uncertainties, and should, therefore, be complemented with time-of-flight (TOF) measurements wherever possible. The final MACSs are compared in Table 1 with the recommended values of Ref. [7], which were based on the previously available results.

\subsection{Implications for the abundance distribution}

The effect of the new cross sections on the abundance distribution of the weak $s$ process was calculated for a stellar model of a $25 M_{\odot}$ star using the post-processing code described in Refs. $[3,12,13]$. The final situation after the shell $\mathrm{C}$ burning phase is compared in Fig. 3 to the results obtained with the previous set of cross sections from Ref. [7]. The reduction in s-process efficiency due to the smaller stellar cross sections of the investigated Fe group nuclei is clearly visible by the significantly reduced abundances between $\mathrm{Zn}$ and $\mathrm{Sr}$.

The range of uncertainties, which are caused by the extrapolation from the measured energy at $k T=25 \mathrm{keV}$ to the higher energies around $k T=90 \mathrm{keV}$ during shell $\mathrm{C}$ burning, are indicated by the thin lines in Fig. 3. To improve this situation further, complementary TOF measurements are clearly needed in the mass region $56 \leq A \leq 70$.

\section{The special case of ${ }^{60} \mathrm{Fe}$}

The origin of ${ }^{60} \mathrm{Fe}$ is related to a variety of astrophysical aspects. The fact that it was found to contribute to Galactic $\gamma$-radioactivity provides crucial information for stellar nucleosynthesis [14, $15,16]$. Long-lived $\gamma$-ray emitters such as ${ }^{60} \mathrm{Fe}$ and ${ }^{26} \mathrm{Al}^{\mathrm{g}}$ are characterized by a diffuse distribution along the galactic plane with the spatial profile of the emission reflecting the Galactic distribution of 


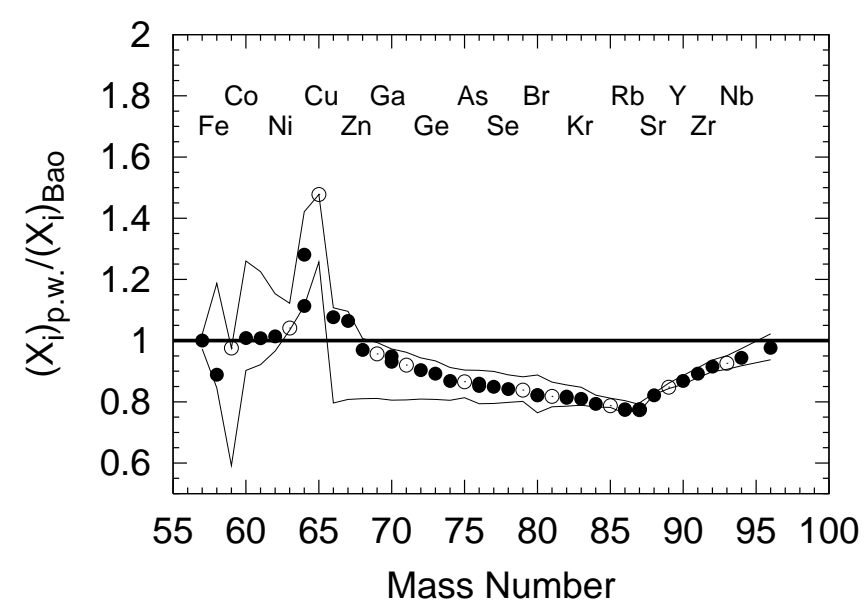

Figure 3: The final $s$-process yields after shell C burning in a $25 M_{\odot}$ star of solar metallicity. To illustrate the combined effect of all new cross sections the yield is plotted relative to the distribution obtained with the previous set of cross sections from Ref. [7] (p.w. stands for present work; even and odd Z elements are distinguished by black and open symbols, respectively). The uncertainties due to the extrapolation of the measured cross sections to higher and lower energies are indicated by the thin solid lines.

a large number of sources. The isotopes ${ }^{26} \mathrm{Al}^{\mathrm{g}}$ and ${ }^{60} \mathrm{Fe}$ are mainly produced in massive stars with under pre-explosive as well as under explosive conditions $[2,17,18]$. In particular, ${ }^{60} \mathrm{Fe}$ receives a significant pre-explosive contribution, being efficiently produced by neutron capture reactions in the high neutron fluxes reached during shell $\mathrm{C}$-burning.

Isotopic anomalies in $\mathrm{Ni}$, in particular an enrichment of ${ }^{60} \mathrm{Ni}$ in meteoritic inclusions [19, 20, $21,22]$, indicate that ${ }^{60} \mathrm{Fe}$ has been present in substantial amounts in the early solar system (ESS), probably injected by a nearby supernova explosion with efficient mixing of the debris with the protosolar nebula $[23,24,25,26]$. In this context, ${ }^{60} \mathrm{Fe}$ represents an important chronometer for the ESS [27].

The recent discovery of ${ }^{60} \mathrm{Fe}$ in deep sea manganese crusts adds another fascinating aspect to this story. The well known growth rate of the crusts implies that fresh ${ }^{60} \mathrm{Fe}$ injection occurred 2.8 Myr ago. This material must have been produced in a nearby massive star and was collected when the solar system swept through its expanding SN envelope. The ${ }^{60} \mathrm{Fe} /{ }^{56} \mathrm{Fe}$ ratio of $2.4 \times 10^{-16}$ measured by accelerator mass spectrometry (AMS) could in principle be used to determine the distance of the $\mathrm{SN}$, an important constraint for the impact of this event on the terrestrial biosphere [28].

As illustrated in Fig. 4, the $s$-process path to ${ }^{60} \mathrm{Fe}$, which starts from the most abundant seed nucleus ${ }^{56} \mathrm{Fe}$, is determined by the branching at ${ }^{59} \mathrm{Fe}\left(t_{1 / 2}=44.5 \mathrm{~d}\right)$. At the low neutron densities during convective core $\mathrm{He}$ burning, ${ }^{60} \mathrm{Fe}$ is shielded from the $s$-process chain, because the $\beta$-decay rate of ${ }^{59} \mathrm{Fe}$ dominates over the $(n, \gamma)$ rate by orders of magnitude. On the other hand, the production of ${ }^{60} \mathrm{Fe}$ becomes efficient during the shell C-burning phase, where higher temperatures of $(1.0-$ 1.4) $\times 10^{9} \mathrm{~K}$ give rise to the neutron densities in excess of $10^{11} \mathrm{~cm}^{-3}$ necessary for bridging the instability gap at ${ }^{59} \mathrm{Fe}$.

The interpretation of the above observations depends critically on the reliability of the stellar 
models as well as on the neutron capture rates for production and depletion of ${ }^{60} \mathrm{Fe}$ [14]. These rates can only be determined reliably in laboratory experiments, because theoretical calculations are too uncertain. Especially in case of ${ }^{60} \mathrm{Fe}$, the calculated values may vary by an order of magnitude [10]. Therefore, the ${ }^{60} \mathrm{Fe}(n, \gamma){ }^{61} \mathrm{Fe}$ cross section has been determined experimentally, using the activation technique described before.

A sample containing $(7.8 \pm 1.6) \times 10^{15}$ atoms of ${ }^{60} \mathrm{Fe}$ was prepared from material extracted from a $\mathrm{Cu}$ beam dump previously irradiated with $590 \mathrm{MeV}$ protons at the Paul-Scherrer-Institute in Switzerland. Besides the desired iron isotope, the original copper sample contained also about 150 $\mathrm{MBq}{ }^{60} \mathrm{Co}$ and $2 \mathrm{MBq}{ }^{44} \mathrm{Ti}$, which have been separated by liquid-liquid extraction with decontamination factors of $\approx 3 \times 10^{8}$ for ${ }^{60} \mathrm{Co}$ and $\approx 5 \times 10^{6}$ for ${ }^{44} \mathrm{Ti}$ [29]. The final diluted $\mathrm{HCl}$ solution contained $100 \mathrm{MBq}$ of ${ }^{55} \mathrm{Fe}$, which is co-produced along with ${ }^{60} \mathrm{Fe}$, as well as traces of stable iron isotopes, originating partly from nuclear reactions and partly from the drilling process. The solution was dried on a graphite backing $6 \mathrm{~mm}$ in diameter to produce the sample for the neutron capture experiment. The number of ${ }^{60} \mathrm{Fe}$ nuclei was determined from the ingrowth of the daughter activity of ${ }^{60} \mathrm{Co}$. Therefore, this value relies on the adopted ${ }^{60} \mathrm{Fe}$ half life of $t_{1 / 2}=1.5 \pm 0.3$, which is given with a rather large uncertainty of $20 \%$ [30].

Because of the short half life of ${ }^{61} \mathrm{Fe}\left(t_{1 / 2}=5.98 \pm 0.06 \mathrm{~min}\right.$ [31] $)$, the experiment was divided into 47 runs. The irradiations lasted for $15 \mathrm{~min}$, followed by a transfer time of about $60 \mathrm{~s}$ from the irradiation position to the counting station, where the activity was measured for $10 \mathrm{~min}$. The subsequent irradiation was started after an additional waiting time of about $15 \mathrm{~min}$.

The induced activities are characterized by $\gamma$-ray lines with known absolute intensities $[31,32]$ and were counted with a pair of HPGe clover detectors, each with a relative efficiency of $120 \%$. By arranging the detectors face to face in very close geometry, absolute peak efficiencies of $56 \%$ and $9.5 \%$ could be achieved at 100 and $1000 \mathrm{keV}$, respectively. The relative position of sample and detectors was exactly defined by a special sample holder to ensure that the counting geometry was reproducible within $\pm 0.1 \mathrm{~mm}$. The detectors were shielded against ambient background by $10 \mathrm{~cm}$ of lead and by an inner layer of $5 \mathrm{~mm}$ copper.

The intrinsic $\gamma$-ray background presented a serious problem for the activity measurements. At low energies, the $\gamma$-spectrum was dominated by internal bremsstrahlung from the decay of the ${ }^{55} \mathrm{Fe}$ impurity, thus impeding the $298 \mathrm{keV}$ line expected to show the best signature for the decay of ${ }^{61} \mathrm{Fe}$. At higher energies, the detection of the stronger lines at 1027 and $1205 \mathrm{keV}$ was difficult due to the lower efficiency and natural background. The background problem could be overcome

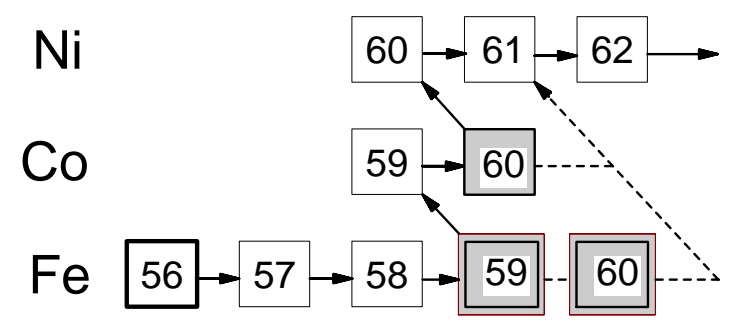

Figure 4: The s-process reaction path to ${ }^{60} \mathrm{Fe}$ via the branching at ${ }^{59} \mathrm{Fe}$. 


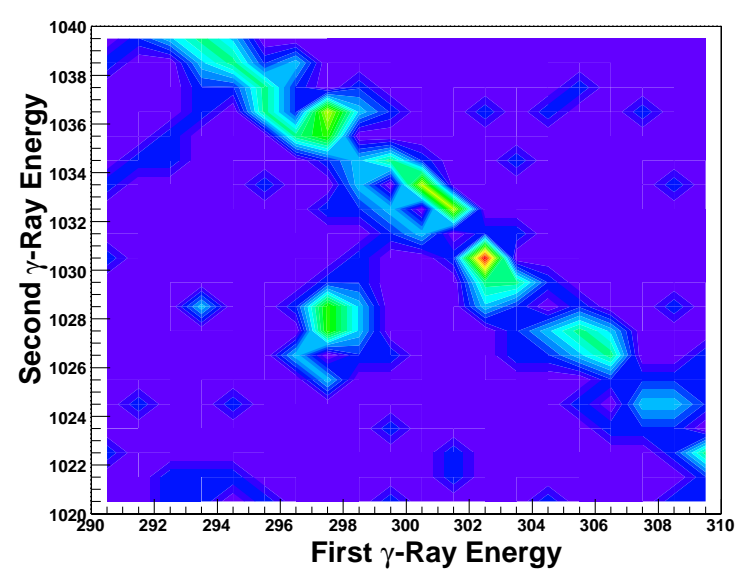

Figure 5: Coincidence spectra of the $298-1027 \mathrm{keV}$ cascades in the decay of ${ }^{61} \mathrm{Fe}$. The diagonal feature corresponds to events with multiplicity 2 originating from Compton scattering of the $1332 \mathrm{keV}{ }^{60} \mathrm{Co}$ line.

by making use of the eight-fold segmentation of the Ge clover array [33]. As shown in Fig. 5 the coincident detection of the $298 / 1027 \mathrm{keV}$ cascade transition provided a nearly background free spectrum, although with reduced counting statistics. Multiplicity cuts helped also to observe the $1205 \mathrm{keV}$ line with lower backgrounds. The activity of the gold foils was counted with the clover array as well, thus reducing the systematic uncertainties of the detection efficiencies.

The final experimental cross section of $10.2 \pm_{1.4}^{2.9}$ (syst) (stat) mbarn was obtained by the sum of all 47 activations. The quoted uncertainty is dominated by the counting statistics $(12 \%)$ and the ${ }^{60} \mathrm{Fe}$ determination (25\%). Since the result is particularly sensitive to the half life of ${ }^{60} \mathrm{Fe}$ used to determine the number of ${ }^{60} \mathrm{Fe}$ atoms, the accuracy of can be signicantly increased once a more accurate half life becomes available.

The extrapolation towards higher thermal energies (Table 2) represents a crucial problem since the statistical model for describing the energy dependence of the ${ }^{60} \mathrm{Fe}$ cross section does not account for the expected resonance structure [10]. Comparison with similar cases in this mass region shows that extrapolation uncertainties may be about $20 \%$ at $k T=90 \mathrm{keV}$, the thermal energy during shell C-burning in massive stars [11].

\section{Summary and outlook}

By a series of new measurements the stellar $(n, \gamma)$ cross sections of several important isotopes in or near the Fe abundance peak has been substantially improved. Since the experiments were

Table 2: Maxwellian Averaged Cross Sections (in mbarn) of ${ }^{60} \mathrm{Fe}(n, \gamma){ }^{61} \mathrm{Fe}$ for $k T=25-100 \mathrm{keV}$

\begin{tabular}{lcccccc}
\hline$k T$ & 25 & 30 & 40 & 60 & 80 & 100 \\
\hline$\langle\sigma \mathrm{v}\rangle / \mathrm{v}_{T}$ & $\mathbf{9 . 9} \pm_{1.4 \text { (stat) }}^{2.8 \text { (syst) }}$ & 9.0 & 7.8 & 6.2 & 5.3 & 4.7 \\
\hline
\end{tabular}


performed by activation in a quasi-stellar neutron spectrum for $k T=25 \mathrm{keV}$, the results need still to be extrapolated to the higher temperatures during the $\mathrm{C}$ shell burning stage corresponding to $k T \approx 90 \mathrm{keV}$ for a more reliable determination of $s$-process nucleosynthesis in massive stars. Therefore, the present results will be complemented by TOF experiments at CERN/n_TOF [34] to determine the energy-dependence of the cross sections with better confidence.

In case of the unstable isotope ${ }^{60} \mathrm{Fe}$, the stellar $(n, \gamma)$ cross section could be measured for the first time. A full discussion of the various observations related to ${ }^{60} \mathrm{Fe}$ would also require an experimental determination of the cross section for the crucial branch point nucleus ${ }^{59} \mathrm{Fe}$. In spite of the severe obstacles due to the short half life of 44 days, the very intense neutron flux at the planned FRANZ facility [35] may nevertheless provide a promising approach for a successful measurement.

\section{Acknowledgements}

We would like to thank S. Köchli and S. Horn for performing the chemical and radiochemical analyses. M.P. acknowledges support from the Marie Curie International Reintegration Grant within the 6th European Community Framework Programme, grant MIRG-CT-2006-046520, and the Joint Institute for Nuclear Astrophysics (JINA) under NSF grant PHY0216783.

\section{References}

[1] T. Rauscher, A. Heger, R. Hoffman, and S. Woosley, Ap. J. 576, 323 (2002).

[2] M. Limongi and A. Chieffi, Ap. J. 647, 483 (2006).

[3] C. Raiteri, M. Busso, R. Gallino, and G. Picchio, Ap. J. 371, 665 (1991).

[4] C. Raiteri et al., Ap. J. 419, 207 (1993).

[5] M. Limongi, O. Straniero, and A. Chieffi, Ap. J. Suppl. 129, 625 (2000).

[6] C. Arlandini et al., Ap. J. 525, 886 (1999).

[7] Z. Bao et al., Atomic Data Nucl. Data Tables 76, 70 (2000).

[8] H. Beer and F. Käppeler, Phys. Rev. C 21, 534 (1980).

[9] W. Ratynski and F. Käppeler, Phys. Rev. C 37, 595 (1988).

[10] T. Rauscher and F.-K. Thielemann, Atomic Data Nucl. Data Tables 75, 1 (2000).

[11] M. Heil et al., Phys. Rev. C 77, 015808 (2008).

[12] C. Raiteri et al., Ap. J. 367, 228 (1991).

[13] M. Pignatari et al., in Nuclei in the Cosmos-IX, http://pos.sissa.it/, edited by A. Mengoni and et al. (Proceedings of Science, SISSA, 2006), p. contribution 061.

[14] R. Diehl, N. Prantzos, and P. von Ballmoos, Nucl. Phys. A777, 70 (2007).

[15] D. Smith, New Astron. Rev. 48, 87 (2004).

[16] M. Harris et al., Astron. Astrophys. 433, L49 (2005).

[17] F. Timmes et al., Ap.J. 449, 204 (1995). 
[18] S. Woosley and A. Heger, Phys. Rep. 442, 269 (2007).

[19] J. Birck and G. Lugmair, Earth Planet. Sci. Lett. 90, 131 (1988).

[20] G. Quitté et al., Ap. J. 655, 678 (2007).

[21] Y. Guan, G. Huss, and L. Leshin, Geochim. Cosmochim. Acta 71, 4082 (2007).

[22] F. Moynier et al., Geochim. Cosmochim. Acta 71, 4365 (2007).

[23] S. Mostefaoui, G. Lugmair, and P. Hoppe, Ap. J. 625, 271 (2005).

[24] L. Looney, J. Tobin, and B. Fields, Ap.J. 632, 1755 (2006).

[25] N. Dauphas et al., Ap. J. (in press).

[26] N. Ouellette, S. Desch, and J. Hester, Ap. J. 662, 1268 (2007).

[27] A. Boss, Ap.J. 660, 1707 (2007).

[28] K. Knie et al., Phys. Rev. Lett. 93, 171103 (2004).

[29] D. Schumann and J. Neuhausen, J. Phys. G: Nucl. Part. Phys. 35, 014046 (2008).

[30] J. Tuli, Nucl. Data Sheets 100, 347 (2003).

[31] M. Bhat, Nucl. Data Sheets 88, 417 (1999).

[32] Z. Chunmei, Nucl. Data Sheets 95, 59 (2002).

[33] R. Reifarth et al., Ap. J. 582, 1251 (2003).

[34] J. Tain, C. Domingo Pardo, F. Bečvář, et al., Technical report, CERN, proposal approved by the CERN INTC February 2006, http://pceet075.cern.ch/proposals/.

[35] U. Ratzinger et al., in Proceedings of the 18th Meeting of the International Collaboration on Advanced Neutron Sources, April 25-29, 2006, Dongguan, Guangdong, PR China p. 210. 